PROCEEDINGS OF THE

AMERICAN MATHEMATICAL SOCIETY

Volume 127, Number 5, Pages 1413-1426

S 0002-9939(99)05049-2

Article electronically published on January 29, 1999

\title{
ESTIMATES FOR THE GREEN FUNCTION OF A GENERAL STURM-LIOUVILLE OPERATOR AND THEIR APPLICATIONS
}

\author{
N. CHERNYAVSKAYA AND L. SHUSTER
}

(Communicated by Hal L. Smith)

\begin{abstract}
For a general Sturm-Liouville operator with nonnegative coefficients, we obtain two-sided estimates for the Green function, sharp by order on the diagonal.
\end{abstract}

\section{INTRODUCTION}

In this paper we study the equation:

$$
-\left(r(x) y^{\prime}(x)\right)^{\prime}+q(x) y(x)=f(x), \quad x \in R .
$$

Here and throughout the paper, $f(x) \in L_{p}(R), \quad p \in[1, \infty)$, and $r(x)$ and $q(x)$ satisfy the following conditions:

$$
\begin{gathered}
r(x)>0, q(x) \geq 0, x \in R ; \quad \frac{1}{r(x)} \in L_{1}^{\mathrm{loc}}(R), q(x) \in L_{1}^{\mathrm{loc}}(R) . \\
\lim _{|d| \rightarrow \infty} \int_{x-d}^{x} \frac{d t}{r(t)} \cdot \int_{x-d}^{x} q(t) d t=\infty, \quad x \in R .
\end{gathered}
$$

Conditions (1.2)-(1.3) guarantee that (1.1) has a unique solution in $L_{p}(R)$ for $p \in$ $[1, \infty)$ (see $\S 2$ ), and under some additional requirement to $r(x)$ and $q(x)$ ([5]; see also $\S 2$ and (1.10) below), the two following assertions hold simultaneously:

1) For any $f(x) \in L_{p}(R)$, equation (1.1) has the unique solution $y(x) \in L_{p}(R)$ :

$$
y(x)=(G f)(x) \stackrel{\text { def }}{=} \int_{-\infty}^{\infty} G(x, t) f(t) d t, \quad x \in R,
$$

$$
\|G\|_{p \rightarrow p}<\infty .
$$

Here (see $\S 2), G(x, t)$ is the Green function corresponding to (1.1)

$$
G(x, t)= \begin{cases}u(x) v(t) & \text { if } \quad x \geq t \\ u(t) v(x) & \text { if } \quad x \leq t\end{cases}
$$

Received by the editors August 21, 1997.

1991 Mathematics Subject Classification. Primary 34B27.

Research of the first author supported by the Israel Academy of Sciences, under Grant 431/95.

Research of the second author supported by the Israel Academy of Sciences, under Grant $505 / 95$.

(C)1999 American Mathematical Society 
and $\{u(x), v(x)\}$ is a special fundamental system of solutions (FSS) of the equation

$$
\left(r(x) z^{\prime}(x)\right)^{\prime}=q(x) z(x), \quad x \in R .
$$

Below, $G(x, t)$ stands for the Green function of a Sturm-Liouville operator $\mathcal{L}$. Here, assuming 1)-2), $\mathcal{L}$ denotes a differential expression $\ell y=-\left(r(x) y^{\prime}(x)\right)^{\prime}+q(x) y(x)$ defined by the solutions of (1.1), while $f(x)$ changes in $L_{p}(R)$ [3]. The goal of the paper is to obtain two-sided estimates for $G(x, t)$. We get such inequalities following the methods suggested in [2], [3]. (In [2], [3] we considered the case $\left.r(x) \equiv 1,1 \leq q(x) \in L_{1}^{\text {loc }}(R)\right)$. In particular, as in [2], [3], we rely on a special formula which gives a representation of $G(x, t)$ via its diagonal values:

$$
G(x, t)=\sqrt{\rho(x) \rho(t)} \exp \left(-\frac{1}{2}\left|\int_{x}^{t} \frac{d \xi}{r(\xi) \rho(\xi)}\right|\right), \rho(x)=\left.G(x, t)\right|_{t=x} .
$$

(In [6], formula (1.8) has been obtained for $r(x) \equiv 1)$. The main advantage of (1.8) compared with (1.6) is the following: under conditions (1.2)-(1.3), in contrast to $\{u(x), v(x)\}$, there have been obtained a priori two-sided estimates for $\rho(x)$ which are sharp by order ([4], [5]; see Theorem 2.2 below):

$$
2^{-1}\left(\int_{x-d_{1}(x)}^{x+d_{2}(x)} q(\xi) d \xi\right)^{-1} \leq \rho(x) \leq 2\left(\int_{x-d_{1}(x)}^{x+d_{2}(x)} q(\xi) d \xi\right)^{-1}, x \in R .
$$

Here, $d_{1,2}(x)$ are auxiliary functions in $r(x), q(x)$ (see $\S 2$ ). Formulae (1.8) and (1.9) immediately lead to estimates of $G(x, t)$ for $x \neq t$ (Theorem 2.2). The obtained inequalities for $G(x, t)$ proves useful for the study of (1.1). As an example of their application, we consider a problem of validity of 1)-2). We show that 1)-2) hold if

$$
\mathcal{A} \stackrel{\text { def }}{=} \inf _{x \in R}\left(\frac{1}{2 d(x)} \int_{x-d(x)}^{x+d(x)} q(\xi) d \xi\right)>0 .
$$

([5]; see $\S 2$, Theorem 2.3 below). Here, $d(x)$ is an auxiliary continuous function, positive for $x \in R$, which can be constructed from $r(x)$ and $q(x)(\S 2)$. In other words, 1)-2) hold if some special average of $q(x)$ (of Steklov type [11]) is separated from zero on the whole number axis. Perhaps condition (1.10) deserves special attention because it is valid for equation (1.1) with oscillating coefficients $q(x)$ (see the example in $\S 2$ ). To conclude, note that Theorem 2.2 proves useful in many other problems related to (1.1). For example, using it one can obtain necessary and sufficient conditions:

(1) for the operator $G: L_{p}(R) \rightarrow L_{p}(R)$ to be compact;

(2) for the operator $\mathcal{L}$ to be coercive;

(3) for solvability of the Neumann and Dirichlet problems for equation (1.1).

These results will be presented in our forthcoming papers.

\section{Statement of Results. Example}

Throughout we denote by $\tau$ absolute positive constants, not essential for exposition, which may differ within a single chain of calculations.

Theorem 2.1. Suppose that one has (1.2) and (2.1):

$$
\int_{-\infty}^{0} q(\xi) d \xi>0, \quad \int_{0}^{\infty} q(\xi) d \xi>0 .
$$


Then there exists a FSS of (1.7) $\{u(x), v(x)\}$ such that

$$
\begin{array}{cc}
u(x)>0, \quad v(x)>0, \quad u^{\prime}(x) \leq 0, \quad v^{\prime}(x) \geq 0 & \text { for } \quad x \in R, \\
r(x)\left[v^{\prime}(x) u(x)-u^{\prime}(x) v(x)\right]=1 & \text { for } \quad x \in R, \\
\lim _{x \rightarrow-\infty} \frac{v(x)}{u(x)}=\lim _{x \rightarrow \infty} \frac{u(x)}{v(x)}=0 . &
\end{array}
$$

Corollary 2.1.1. Suppose that one has (1.2) and (2.1). Then (1.7) has no solutions $z(x) \in L_{p}(R)$ apart from $z(x) \equiv 0$.

Definition 2.1. An FSS of (1.6) satisfying (2.2) is called a principal FSS (PFSS).

Lemma 2.1. For $\{u(x), v(x)\}$ one has the following representations:

$$
\begin{aligned}
& u(x)=\sqrt{\rho(x)} \exp \left(-\frac{1}{2} \int_{x_{1}}^{x} \frac{d \xi}{r(\xi) \rho(\xi)}\right), \\
& v(x)=\sqrt{\rho(x)} \exp \left(\frac{1}{2} \int_{x_{1}}^{x} \frac{d \xi}{r(\xi) \rho(\xi)}\right)
\end{aligned}
$$

where $x \in R, \quad \rho(x)=u(x) v(x), x_{1}$ is the unique solution of the equation $u(x)=$ $v(x)$ in $R$. Moreover, for $G(x, t)$ and $\rho(x)$ one has representation (1.8) and (2.4):

$$
\int_{-\infty}^{0} \frac{d t}{r(t) \rho(t)}=\int_{0}^{\infty} \frac{d t}{r(t) \rho(t)}=\infty
$$

Formulae (2.3) were applied in $[13, \S 19.53]$, [4], [5], and, for $r(x) \equiv 1$, in [2], [3], [6].

Lemma 2.2. For every fixed $x \in R$, each of the equations in $d \geq 0$ :

$$
1=\int_{x-d}^{x} \frac{d t}{r(t)} \cdot \int_{x-d}^{x} q(t) d t, \quad 1=\int_{x}^{x+d} \frac{d t}{r(t)} \cdot \int_{x}^{x+d} q(t) d t
$$

has a unique finite positive solution.

Denote the solutions of $(2.5)$ by $d_{1}(x), d_{2}(x)$, respectively. For $x \in R$ let us introduce the following functions:

$$
\begin{aligned}
& \varphi(x)=\int_{x-d_{1}(x)}^{x} \frac{d t}{r(t)}, \quad \psi(x)=\int_{x}^{x+d_{2}(x)} \frac{d t}{r(t)}, \\
& h(x)=\frac{\varphi(x) \psi(x)}{\varphi(x)+\psi(x)} \equiv\left(\int_{x-d_{1}(x)}^{x+d_{2}(x)} q(\xi) d \xi\right)^{-1} .
\end{aligned}
$$

Theorem 2.2. For $x, t \in R$ one has inequalities:

$$
\begin{gathered}
2^{-1} v(x) \leq\left(r(x) v^{\prime}(x)\right) \varphi(x) \leq 2 v(x) \\
2^{-1} u(x) \leq\left(r(x)\left|u^{\prime}(x)\right|\right) \psi(x) \leq 2 u(x) \\
2^{-1} h(x) \leq \rho(x) \leq 2 h(x), \\
2^{-1} \exp \left(-\left|\int_{x}^{t} \frac{d \xi}{r(\xi) h(\xi)}\right|\right) \leq \frac{G(x, t)}{\sqrt{h(x) h(t)}} \leq 2 \exp \left(-\frac{1}{4}\left|\int_{x}^{t} \frac{d \xi}{r(\xi) h(\xi)}\right|\right) .
\end{gathered}
$$


Corollary 2.2.1. Suppose that one has (1.2)-(1.3) and (2.10):

$$
a^{-1} \leq r(x) \leq a \quad \text { for } x \in R, \quad a=\text { const, } a \in[1, \infty) .
$$

Consider the following equation in $d \geq 0$ for a fixed $x \in R$ :

$$
S(d)=2, \quad S(d) \stackrel{\text { def }}{=} d \int_{x-d}^{x+d} q(\xi) d \xi .
$$

Equation (2.11) has a unique finite positive solution for every $x \in R$. If $\tilde{d}(x)$ is the solution of (2.11), then the following estimates hold:

$$
(2 a+2)^{-1} \tilde{d}(x) \leq \rho(x) \leq 2^{-1}(2 a+1) \tilde{d}(x), \quad x \in R .
$$

Remark. The function $\tilde{d}(x)$ has been introduced by M. Otelbaev ([7]). Estimates (2.7)-(2.8) are given in [4], [5].

For $q(x) \geq \varepsilon>0, x \in R$, estimates (2.7)-(2.8) with other, more complicated auxiliary functions were obtained in [8]. The method in [8] does not allow us to remove the restriction $q(x) \geq \varepsilon>0$ because it uses division by $q(x)$. Our method can be viewed as a development of [1], [3], [10]. See [5, §2] for a review of the results of type (2.7)-(2.8).

Lemma 2.3. For every fixed $x \in R$, the equation in $d \geq 0$ :

$$
1=\int_{x-d}^{x+d} \frac{d t}{r(t) h(t)}
$$

has a unique finite positive solution. Denote this solution by $d(x)$. The function $d(x)$ is continuous for $x \in R$.

Theorem 2.3. If $\mathcal{A}>0$ (see (1.10)), $p \in[1, \infty)$, then assertions 1)-2), in $\S 1$, are valid and

$$
\left\|q(x)^{1 / p} y(x)\right\|_{p} \leq(\tau \mathcal{A})^{-1 / p^{\prime}}\|f\|_{p} .
$$

Corollary 2.3.1. Let $f(x) \in L_{1}(R)$. If $\mathcal{A}>0$, then (1.1) has the unique solution $y(x)$ in $L_{1}(R)$, (1.4)-(1.5) hold, and, moreover,

$$
\left\|\left(r(x) y^{\prime}(x)\right)^{\prime}\right\|_{1}+\|q(x) y(x)\|_{1} \leq 3\|f(x)\|_{1} .
$$

Remark. Corollary 2.3.1 generalizes the corresponding results of [8].

Usually one is not able to calculate exact values of $\varphi(x), \psi(x), d(x)$. However, to apply Theorems $2.2-2.3$, it is sufficient to have two-sided estimates of these functions.

The following assertion gives a method for obtaining such estimates. This theorem is technical, and we give it without proof. See [4], [5] for details.

Theorem 2.4. Suppose that (1.2) holds and there are continuous positive functions $r_{1}(x), q_{1}(x)$ and functions $r_{2}(x), q_{2}(x)$ such that

$$
r(x)=r_{1}(x)+r_{2}(x), \quad q(x)=q_{1}(x)+q_{2}(x), \quad x \in R ;
$$

2) there are constants $a \geq 1, b>0$ such that for $|x| \gg 1$ one has the inequalities

$$
\frac{1}{a} \leq \frac{r_{1}(t)}{r_{1}(x)}, \frac{q_{1}(t)}{q_{1}(x)} \leq a \quad \text { for } \quad|t-x| \leq b \hat{d}(x), \hat{d}(x)=\sqrt{\frac{r_{1}(x)}{q_{1}(x)}}
$$


3) there is a constant $\delta \in(0,1]$ such that $r(x) \geq \delta r_{1}(x)$ for $x \in R$;

4) $\varkappa_{1}(x) \rightarrow 0, \varkappa_{2}(x) \rightarrow 0$ as $|x| \rightarrow \infty$, where

$$
\begin{aligned}
& \varkappa_{1}(x)=\frac{1}{\sqrt{r_{1}(x) q_{1}(x)}} \sup _{|z| \leq b \hat{d}(x)}\left|\int_{x}^{x+z} q_{2}(t) d t\right|, \\
& \varkappa_{2}(x)=\sqrt{r_{1}(x) q_{1}(x)} \sup _{|z| \leq b \hat{d}(x)}\left|\int_{x}^{x+z} \frac{r_{2}(t)}{r_{1}(t)^{2}} d t\right| .
\end{aligned}
$$

Then the following assertions hold:

A) If conditions 1)-4) are satisfied and $b \geq 3 a$, then (1.3) holds. In addition,

$$
\begin{array}{ll}
\tau^{-1}\left(r_{1}(x) q_{1}(x)\right)^{-1 / 2} \leq \varphi(x), \psi(x) \leq \tau\left(r_{1}(x) q_{1}(x)\right)^{-1 / 2}, & |x| \gg 1 \\
\tau^{-1}\left(r_{1}(x) q_{1}(x)\right)^{-1 / 2} \leq h(x) \leq \tau\left(r_{1}(x) q_{1}(x)\right)^{-1 / 2}, & x \in R .
\end{array}
$$

B) Under the hypotheses of $\mathrm{A})$, if $r(x) \leq \tau r_{1}(x)$, then

$$
\begin{aligned}
& \frac{\tau^{-1}}{\sqrt[4]{r_{1}(t) r_{1}(x) q_{1}(t) q_{1}(x)}} \exp \left(-\tau\left|\int_{x}^{t} \sqrt{\frac{q_{1}(\xi)}{r_{1}(\xi)}} d \xi\right|\right) \leq G(x, t) \\
& \leq \frac{\tau}{\sqrt[4]{r_{1}(t) r_{1}(x) q_{1}(t) q_{1}(x)}} \exp \left(-\frac{1}{\tau}\left|\int_{x}^{t} \sqrt{\frac{q_{1}(\xi)}{r_{1}(\xi)}} d \xi\right|\right), \quad x, t \in R .
\end{aligned}
$$

C) If conditions 1)-4) are satisfied, and $b \geq 160 a^{3} \delta^{-2}$, then (see (2.17)):

$$
\tau^{-1} \hat{d}(x) \leq d(x) \leq \tau \hat{d}(x), \quad x \in R .
$$

D) If, in addition, the function $q(x)$ is such that for any $\alpha>0$ and $x \in R$ one has the inequality $\int_{x-\alpha}^{x+\alpha} q(\xi) d \xi>0$, then $\mathcal{A}>0$ (see (1.10)) if and only if $\inf _{x \in R} q_{1}(x)>0$.

Example. Consider equation (1.1) with the coefficients for $x \in R$ :

$$
r(x)=\left(1+x^{2}\right)^{-1}+2^{-1}\left(1+x^{2}\right)^{-1} \cos \left(e^{|x|}\right), \quad q(x)=2 e^{|x|} \cos ^{2}\left(e^{|x|}\right) .
$$

According to Theorem 2.4, choose $r_{1}(x)=\left(1+x^{2}\right)^{-1}, q_{1}(x)=e^{|x|}$. Then (see (2.17)) $\hat{d}(x)=\left(1+x^{2}\right)^{-1 / 2} e^{-|x| / 2} \rightarrow 0$ as $|x| \rightarrow \infty$. Let us verify that for any fixed $b>0$ all the hypotheses of Theorem 2.3 are satisfied. One can assume $x \geq 0$ because all the above functions are even. Since $\hat{d}(x) \rightarrow 0$ as $x \rightarrow \infty$ and

$$
\frac{r_{1}(x)}{r_{1}(t)}=1+(t-x) \frac{t+x}{1+x^{2}}, \quad \frac{q_{1}(t)}{q_{1}(x)}=e^{t-x},
$$

one can see that 2) holds, say for $a=5 / 4$. Clearly in this case, $\delta=1 / 2$ and 3) also holds. Further,

$$
\varkappa_{1}(x)=\frac{\sqrt{1+x^{2}}}{e^{x / 2}} \sup _{|z| \leq b \hat{d}(x)}\left|\int_{x}^{x+z} e^{t} \cos \left(e^{t}\right) d t\right| \leq \frac{2 \sqrt{1+x^{2}}}{e^{x / 2}} .
$$


To estimate $\varkappa_{2}(x)$, we use the second mean theorem $[12$, Ch.12, $\S 3]$ which can be applied here for $x \gg 1$ :

$$
\begin{aligned}
& \varkappa_{2}(x)=\frac{1}{2} \frac{e^{x / 2}}{\sqrt{1+x^{2}}} \sup _{|z| \leq b \hat{d}(x)}\left|\int_{x}^{x+z}\left[\frac{1+t^{2}}{e^{t}}\right]\left[e^{t} \cos e^{t}\right] d t\right| \\
& \leq \frac{\tau e^{x / 2}}{\sqrt{1+x^{2}}} \sup _{|z| \leq b \hat{d}(x)} \frac{1+x^{2}}{e^{x}}\left|\int_{x}^{x+z} e^{t} \cos e^{t} d t\right| \leq \tau \frac{\sqrt{1+x^{2}}}{e^{x / 2}}
\end{aligned}
$$

From the estimates for $\varkappa_{1,2}(x)$, it follows that all the hypotheses of Theorem 2.4, including A), B), C), are satisfied. Hence

$$
\begin{gathered}
\tau^{-1} \sqrt{1+x^{2}} e^{-|x| / 2} \leq \rho(x) \leq \tau \sqrt{1+x^{2}} e^{-|x| / 2}, \quad x \in R, \\
G(x, t) \leq \tau \sqrt[4]{\left(1+t^{2}\right)\left(1+x^{2}\right)} e^{-\frac{|x|+|t|}{4}} \exp \left(-\frac{1}{\tau}\left|\int_{x}^{t} \sqrt{\left(1+\xi^{2}\right)} e^{\xi / 2} d \xi\right|\right), x, t \in R .
\end{gathered}
$$

From the graph of $q(x)$ one can see that D) also holds, and $q_{1}(x) \geq 1$ for $x \in R$. Hence, by Theorem 2.3 for (1.1) with coefficients (2.23), one has assertions 1)-2) of $\S 1$.

Remark. From the proofs given below it follows that one can replace condition (1.3) with a weaker one:

$$
2 \mu \stackrel{\text { def }}{=} \inf _{x \in R} \lim _{|d| \rightarrow \infty}\left(\int_{x-d}^{x} \frac{d t}{r(t)} \cdot \int_{x-d}^{x} q(t) d t\right)>0
$$

without essentially affecting the main results of the paper. In particular, if $\mu<\infty$, then to obtain estimates for $\rho(x)$ and $G(t, x)$, one has to consider the equations

$$
\mu=\int_{x-d}^{x} \frac{d t}{r(t)} \int_{x-d}^{x} q(t) d t, \quad \mu=\int_{x}^{x+d} \frac{d t}{r(t)} \int_{x}^{x+d} q(t) d t
$$

instead of (2.5), and then to repeat the proofs from $\S 3-\S 5$.

\section{Construction of a PFSS and its properties}

In this section we prove Theorem 2.1. We need the following lemma.

Lemma 3.1. Suppose that (1.2) and (2.1) hold. Then the solution $z(x)$ of the Cauchy problem for (1.7) with $z(0)=1, z^{\prime}(0)=0$, satisfies the following relations:

$$
z(x) \geq 1, \quad \operatorname{sign} z^{\prime}(x)=\operatorname{sign} x \text { for } x \in R, \quad c_{0} \stackrel{\text { def }}{=} \int_{-\infty}^{\infty} \frac{d \xi}{r(\xi) z^{2}(\xi)}<\infty .
$$

Proof. Let us verify that $z(x)>0$ for $x \in R_{+}$. Since $z(0)=1$, there is a neighborhood of zero where $z(x)>0$. Suppose that $z(x)$ has zeroes on $x \in R_{+}$, and let $x_{0}>0$ be the first zero of $z(x)$. Then $z^{\prime}\left(x_{0}\right) \leq 0$. Indeed, otherwise $z(t)<0$ for $t<x_{0}$, provided $t$ is sufficiently close to $x_{0}$. But then there is a root of $z(x)$ on $\left(0, x_{0}\right)$, a contradiction to the choice of $x_{0}$. On the other hand,

$$
r\left(x_{0}\right) z^{\prime}\left(x_{0}\right)=\int_{0}^{x_{0}} q(\xi) z(\xi) d \xi \geq 0 .
$$

Hence $z\left(x_{0}\right)=z^{\prime}\left(x_{0}\right)=0$, i.e., $z(x) \equiv 0$ for $x \in R$, a contradiction to $z(0)=1$. Thus, for $x \in R_{+}$one has $z(x)>0 \Rightarrow z^{\prime}(x) \geq 0 \Rightarrow z(x) \geq 1, x \geq 0$. From (1.2) and 
(2.1) it follows that there exists $\tau \gg 1$ such that $\int_{0}^{\tau} q(\xi) d \xi \geq \tau^{-1}$. By the above proof we get

$$
r(\tau) z^{\prime}(\tau)=\int_{0}^{\tau} q(\xi) z(\xi) d(\xi) \geq \int_{0}^{\tau} q(\xi) d \xi \geq \frac{1}{\tau} .
$$

Since $r(x) z^{\prime}(x)$ is a nondecreasing function, then $r(x) z^{\prime}(x) \geq r(\tau) z^{\prime}(\tau) \geq \tau^{-1}$ for $x \geq \tau$. Hence

$$
z(x) \geq z(\tau)+\frac{1}{\tau} \int_{\tau}^{x} \frac{d \xi}{r(\xi)} \geq 1+\frac{1}{\tau} \int_{\tau}^{x} \frac{d \xi}{r(\xi)}, \quad x \geq \tau .
$$

From the latter estimate it follows that

$$
\begin{aligned}
\int_{\tau}^{\infty} \frac{d \xi}{r(\xi) z^{2}(\xi)} & \leq \int_{\tau}^{\infty} \frac{1}{r(\xi)}\left[1+\frac{1}{\tau} \int_{\tau}^{\xi} \frac{d s}{r(s)}\right]^{-2} d \xi \\
& =\tau\left\{1-\left[1+\frac{1}{\tau} \int_{\tau}^{\infty} \frac{d s}{r(s)}\right]^{-1}\right\} \leq \tau .
\end{aligned}
$$

Clearly, $\left(r(x) z^{2}(x)\right)^{-1} \in L_{1}(0, \tau)$. Thus, $\left(r(x) z(x)^{2}\right)^{-1} \in L_{1}\left(R_{+}\right)$. One can similarly check the other statements of the lemma.

Proof of Theorem 2.1. Let $z(x)$ be the solution $z(x)$ from Lemma 3.1. Set

$$
v(x)=\frac{z(x)}{\sqrt{c_{0}}} \int_{-\infty}^{x} \frac{d \xi}{r(\xi) z^{2}(\xi)}, \quad u(x)=\frac{z(x)}{\sqrt{c_{0}}} \int_{x}^{\infty} \frac{d \xi}{r(\xi) z^{2}(\xi)}, \quad x \in R
$$

where $c_{0}$ is defined by (3.1). The functions $\{u(x), v(x)\}$, form a PFSS of (1.7). Let us establish (2.2). Verify that $u^{\prime}(x) \leq 0$ for $x \in R$. Indeed, since by (3.1),

$$
\sqrt{c_{0}} u^{\prime}(x)=z^{\prime}(x) \int_{x}^{\infty} \frac{d \xi}{r(\xi) z^{2}(\xi)}-\frac{1}{r(x) z(x)}, \quad x \in R,
$$

we have $u^{\prime}(x) \leq 0$ for $x \leq 0$. Let $x>0$. Since $r(x) z^{\prime}(x)$ does not decrease, then

$$
\begin{aligned}
\sqrt{c_{0}} u^{\prime}(x) & =z^{\prime}(x) \int_{x}^{\infty} \frac{d t}{r(t) z^{2}(t)}-\frac{1}{r(x) z(x)} \leq \frac{1}{r(x)} \int_{x}^{\infty} \frac{z^{\prime}(t) d t}{z^{2}(t)}-\frac{1}{r(x) z(x)} \\
& =-\frac{1}{r(x) z(\infty)} \leq 0 .
\end{aligned}
$$

The remaining relations in (2.2) are either obvious or can be similarly checked.

Proof of Corollary 2.1.1. Assume the contrary. Then for some $\tau_{1}, \tau_{2}\left(\left|\tau_{1}\right|+\left|\tau_{2}\right|\right.$ $\neq 0)$, one has $z(x)=\tau_{1} u(x)+\tau_{2} v(x) \in L_{p}(R)$. For example, let $\tau_{2} \neq 0$. By $(2.2)$ there is $\tau>0$ such that $u(x) v(x)^{-1}\left|\tau_{1} \tau_{2}^{-1}\right| \leq 2^{-1}$ for $x \geq \tau$. Then

$$
\|z(x)\|_{p}^{p}>\int_{\tau}^{\infty}\left|\tau_{1} u(t)+\tau_{2} v(t)\right|^{p} d t \geq\left|\tau_{2} v(\tau)\right|^{p} \int_{\tau}^{\infty}|1-| \frac{\tau_{1}}{\tau_{2}} \frac{u(t)}{v(t)}||^{p} d t=\infty .
$$

Hence $\tau_{2}=0$. Since $u(x) \notin L_{p}(-\infty, 0)$, one also has $\tau_{1}=0$.

Remark. This method of construction of a PFSS was used in [9]. 


\section{Estimates of the Green function}

In this section we prove Theorem 2.2 and Corollary 2.2.1.

Proof of Lemma 2.1. Let $F(x)=v(x)-u(x)$. Then $F^{\prime}(x)=v^{\prime}(x)-u^{\prime}(x) \geq 0$ for $x \in R$ (see (2.2)). From (3.2) it follows that $F(x)<0$ as $x \rightarrow-\infty, F(x)>0$ as $x \rightarrow \infty$. Then there is a unique root $x_{1}$ to $F(x)=0$. Since

$$
\frac{v^{\prime}(t)}{v(t)}-\frac{u^{\prime}(t)}{u(t)}=\frac{1}{r(t) u(t) v(t)}=\frac{1}{r(t) \rho(t)}, \quad t \in R
$$

(see $(2.2)$ ), from (4.1) it follows that $\{u(x), v(x)\}$ are solutions to the system

$$
\begin{cases}\frac{v(x)}{u(x)}=\exp \left(\int_{x_{1}}^{x} \frac{d t}{r(t) \rho(t)}\right) & x \in R \\ u(x) v(x)=\rho(x) & x \in R .\end{cases}
$$

Solving (4.2) with respect to $v(x), u(x)$ gives (2.3). To obtain (1.8), one has to substitute (2.3) into (1.6).

Corollary 2.1.1. To obtain (2.4), one has to substitute (2.3) into (2.2).

Proof of Lemma 2.2. Clearly, $\Phi_{1}(0)=\Phi_{2}(0)=0, \quad \Phi_{1}(\infty)=\Phi_{2}(\infty)=\infty$, where

$$
\Phi_{1}(d)=\int_{x-d}^{x} \frac{d t}{r(t)} \int_{x-d}^{x} q(t) d t, \quad \Phi_{2}(d)=\int_{x}^{x+d} \frac{d t}{r(t)} \int_{x}^{x+d} q(t) d t, \quad d \geq 0 .
$$

The functions $\Phi_{1,2}(d)$ do not decrease on $[0, \infty)$. Then the equations $\Phi_{1}(d)=$ 1, $\Phi_{2}(d)=1$ have unique finite positive solutions.

Proof of Theorem 2.2. We use the method of [1], [3]. Let us check (2.7) for $v(x)$. (One can get the estimates for $u(x)$ similarly.) Let us integrate the equation $\left(r(\xi) v^{\prime}(\xi)\right)^{\prime}=q(\xi) v(\xi), \quad \xi \in R$ along $[x-t, x], t \geq 0$, divide the obtained integral by $r(x-t)$ and integrate the result by $t \in[0, s], s \geq 0$; we finally obtain

$$
\begin{aligned}
r(x) v^{\prime}(x) \int_{x-s}^{x} \frac{d \xi}{r(\xi)}= & v(x)-v(x-s) \\
& +\int_{x-s}^{x} \frac{1}{r(t)} \int_{t}^{x} q(\xi) v(\xi) d \xi d t, x \in R, s \geq 0 .
\end{aligned}
$$

In (4.3) set $s=d_{1}(x)$ (see (2.5)). Then from (2.2), (2.6) and (2.5) we obtain

$$
r(x) v^{\prime}(x) \varphi(x)<v(x)+v(x) \int_{x-d_{1}(x)}^{x} \frac{d t}{r(t)} \int_{x-d_{1}(x)}^{x} q(t) d t=2 v(x), \quad x \in R .
$$

The right hand side of (2.7) is checked. Furthermore, from (4.3) it follows that

$$
v(x) \leq v(x-s)+r(x) v^{\prime}(x) \int_{x-s}^{x} \frac{d \xi}{r(\xi)}, \quad x \in R, \quad s \geq 0
$$


Let us multiply (4.4) by $q(x-s)$, integrate the result by $s \in\left[0, d_{1}(x)\right]$, and use (2.2), (2.6) and (2.5) again:

$$
\begin{aligned}
& \frac{v(x)}{\varphi(x)}=v(x) \int_{x-d_{1}(x)}^{x} q(t) d t=v(x) \int_{0}^{d_{1}(x)} q(x-s) d s \\
& \leq \int_{0}^{d_{1}(x)} q(x-s) v(x-s) d s+r(x) v^{\prime}(x) \int_{0}^{d_{1}(x)} q(x-s) \int_{x-s}^{x} \frac{d \xi}{r(\xi)} d s \\
& \leq \int_{x-d_{1}(x)}^{x}\left(r(\xi) v^{\prime}(\xi)\right)^{\prime} d \xi+r(x) v^{\prime}(x) \int_{x-d_{1}(x)}^{x} q(t) d t \int_{x-d_{1}(x)}^{x} \frac{d t}{r(t)}<2 r(x) v^{\prime}(x) .
\end{aligned}
$$

Thus we have proved (2.7). Further, as in [3], by (2.6), (2.2) and (2.7) we obtain (2.8):

$$
\begin{aligned}
& \frac{\rho(x)}{h(x)}=\frac{\frac{1}{\varphi(x)}+\frac{1}{\psi(x)}}{\frac{r(x) v^{\prime}(x)}{v(x)}+\frac{r(x)\left|u^{\prime}(x)\right|}{u(x)}} \leq \max \left\{\frac{v(x)}{r(x) v^{\prime}(x)} \frac{1}{\varphi(x)}, \frac{u(x)}{r(x)\left|u^{\prime}(x)\right|} \frac{1}{\psi(x)}\right\} \leq 2, \\
& \frac{\rho(x)}{h(x)}=\frac{\frac{1}{\varphi(x)}+\frac{1}{\psi(x)}}{\frac{r(x) v^{\prime}(x)}{v(x)}+\frac{r(x)\left|u^{\prime}(x)\right|}{u(x)}} \geq \min \left\{\frac{v(x)}{r(x) v^{\prime}(x)} \frac{1}{\varphi(x)}, \frac{u(x)}{r(x)\left|u^{\prime}(x)\right|} \frac{1}{\psi(x)}\right\} \geq \frac{1}{2} .
\end{aligned}
$$

From (2.8) and (1.8) one deduces (2.9).

Proof of Corollary 2.2.1. From (1.2), (1.3) and (2.10) it follows that for $d \gg 1$, one has

$$
a d \int_{x-d}^{x} q(\xi) d \xi>1, a d \int_{x}^{x+d} q(\xi) d \xi>1, \text { ad } \int_{x-d}^{x+d} q(\xi) d \xi>2, \quad x \in R .
$$

For a fixed $x \in R$ consider the function $S(d), d \geq 0$ (see (2.11)). From (2.11) and (4.6) it follows that $S(0)=0, S(\infty)=\infty$. Since $S(d)$ does not decrease on $R_{+}$, there exists a unique solution $\tilde{d}(x)$ to the equation $S(d)=2$. Further, one has an analogue of (4.3) for $u(x)$ for $s \geq 0$ :

$$
\begin{aligned}
r(x)\left|u^{\prime}(x)\right| \int_{x}^{x+s} \frac{d t}{r(t)}= & u(x)-u(x+s) \\
& +\int_{x}^{x+s} \frac{1}{r(t)} \int_{x}^{t} q(\xi) u(\xi) d \xi d t, \quad x \in R .
\end{aligned}
$$

In (4.3), (4.7) set $s=\tilde{d}(x)$. Then by (2.2), (2.10), and the definition of $\tilde{d}(x)$, one has

$$
\begin{aligned}
\frac{1}{\rho(x)} & =\frac{r(x) v^{\prime}(x)}{v(x)}+\frac{r(x)\left|u^{\prime}(x)\right|}{u(x)}<\left(\int_{x-\tilde{d}(x)}^{x} \frac{d t}{r(t)}\right)^{-1}+\left(\int_{x}^{x+\tilde{d}(x)} \frac{d t}{r(t)}\right)^{-1} \\
& +\int_{x-\tilde{d}(x)}^{x+\tilde{d}(x)} q(t) d t \leq \frac{2+2 a}{\tilde{d}(x)} .
\end{aligned}
$$


Let us proceed as in the proof of (4.5), but integrate by $s \in[0, \tilde{d}(x)]$. We obtain

$$
\begin{aligned}
& v(x) \int_{x-\tilde{d}(x)}^{x} q(t) d t<r(x) v^{\prime}(x)\left[1+\int_{x-\tilde{d}(x)}^{x} \frac{d t}{r(t)} \cdot \int_{x-\tilde{d}(x)}^{x} q(t) d t\right] \\
& \leq r(x) v^{\prime}(x)\left[1+a \tilde{d}(x) \int_{x-\tilde{d}(x)}^{x} q(t) d t\right], \\
& u(x) \int_{x}^{x+\tilde{d}(x)} q(t) d t<r(x)\left|u^{\prime}(x)\right|\left[1+\int_{x}^{x+\tilde{d}(x)} q(t) d t \cdot \int_{x}^{x+\tilde{d}(x)} \frac{d t}{r(t)}\right] \\
& \leq r(x)\left|u^{\prime}(x)\right|\left[1+a \tilde{d}(x) \int_{x}^{x+\tilde{d}(x)} q(t) d t\right] .
\end{aligned}
$$

These inequalities, the definition of $\tilde{d}(x)$ and (2.2) imply

$$
\begin{aligned}
\frac{2}{\tilde{d}(x)} & =\int_{x-\tilde{d}(x)}^{x+\tilde{d}(x)} q(t) d t \\
& <\left[\frac{r(x) v^{\prime}(x)}{v(x)}+\frac{r(x)\left|u^{\prime}(x)\right|}{u(x)}\right]\left[1+a \tilde{d}(x) \int_{x-\tilde{d}(x)}^{x+\tilde{d}(x)} q(t) d t\right]=\frac{2 a+1}{\rho(x)} .
\end{aligned}
$$

5. InVERsion of A NON-HOMOgeneous Sturm-Liouville EQUATion in $L_{p}(R)$

In this section we prove Theorem 2.3.

Proof of Lemma 2.3. Clearly, $2 \Phi_{1}(d) \geq \Phi_{2}(d)$ for $d \geq 0$, where

$$
\Phi_{1}(d)=\int_{x-d}^{x+d} \frac{d t}{r(t) h(t)}, \quad \Phi_{2}(d)=\int_{x-d}^{x+d} \frac{d t}{r(t) \rho(t)}, \quad x \in R
$$

(see (2.8)). Since by (2.4) one has $\Phi_{2}(\infty)=\infty$, we obtain $\Phi_{1}(0)=0, \Phi_{1}(\infty)=\infty$ and $\Phi_{1}(d)$ is monotone increasing on $(0, \infty)$. Therefore, the equation $\Phi_{1}(d)=1$ has a unique, finite positive solution. Let $d(x)$ be this solution. Then,

$$
|d(x+s)-d(x)| \leq|s| \quad \text { for } \quad|s| \leq d(x), x \in R .
$$

Indeed, let $s \in[0, d(x)]$. Clearly,

$$
1 \leq \int_{(x+s)-(d(x)+s)}^{(x+s)+(s+d(x))} \frac{d \xi}{r(\xi) h(\xi)}, \quad 1 \geq \int_{(x+s)-(d(x)-s)}^{(x+s)+(d(x)-s)} \frac{d \xi}{r(\xi) h(\xi)} .
$$

By the definition of $d(x)$, taking into account that $\Phi_{1}(d)$ is monotone, these inequalities imply respectively $d(x+s) \leq d(x)+s, d(x+s) \geq d(x)-s$, i.e., (5.1) holds. The case $s \in[-d(x), 0]$ can be treated similarly. By $(5.1), d(x)$ is continuous.

Lemma 5.1. Let $x \in R, t \in[x-d(x), x+d(x)]$. Then

$$
\begin{array}{ll}
\alpha^{-1} v(x) \leq v(t) \leq \alpha v(x), & \alpha^{-1} u(x) \leq u(t) \leq \alpha u(x), \quad \alpha=\exp (2), \\
\alpha^{-1} \rho(x) \leq \rho(t) \leq \alpha \rho(x), & (4 \alpha)^{-1} h(x) \leq h(t) \leq(4 \alpha) h(x) .
\end{array}
$$


Proof. For $t \in[x, x+d(x)]$ from (2.7) and (2.6), it follows that

$$
\ln \frac{v(t)}{v(x)} \leq 2 \int_{x}^{t} \frac{d \xi}{r(\xi) \varphi(\xi)}<2 \int_{x}^{t} \frac{d \xi}{r(\xi) h(\xi)}<2 \int_{x-d(x)}^{x+d(x)} \frac{d \xi}{r(\xi) h(\xi)}=2 .
$$

For $t \in[x-d(x), x]$ we similarly get

$$
\ln \frac{v(x)}{v(t)} \leq 2 \int_{t}^{x} \frac{d \xi}{r(\xi) \varphi(\xi)} \leq 2 \int_{t}^{x} \frac{d \xi}{r(\xi) h(\xi)}<2 \int_{x-d(x)}^{x+d(x)} \frac{d \xi}{r(\xi) h(\xi)}=2 .
$$

These inequalities and (2.2) imply (5.2) for $v(x)$. For $u(x)$ one can check (5.2) in a similar way. Further, from (2.2) and (5.2) we obtain

$$
\begin{array}{ll}
\frac{p(t)}{p(x)}=\frac{u(t)}{u(x)} \cdot \frac{v(t)}{v(x)} \leq \frac{v(t)}{v(x)} \leq \alpha & \text { for } \quad t \in[x, x+d(x)], \\
\frac{p(t)}{p(x)}=\frac{u(t)}{u(x)} \cdot \frac{v(t)}{v(x)} \geq \frac{u(t)}{u(x)} \geq \alpha^{-1} & \text { for } \quad t \in[x, x+d(x)] .
\end{array}
$$

For $t \in[x-d(x), x]$ estimates (5.3) for $\rho(\cdot)$ can be obtained similarly. Estimates (5.3) for $h(x)$ follow from estimates (5.4) for $\rho(x)$ and (2.8).

Definition 5.1. We say that a system of segments $\left\{\Delta_{n}\right\}_{n \in N^{\prime}}, N^{\prime}=\{ \pm 1, \pm 2, \ldots\}$ forms an $R(x)$-covering of $R$ if the following assertions hold:

(1) $\Delta_{n}=\left[\Delta_{n}^{-}, \Delta_{n}^{+}\right] \stackrel{\text { def }}{=}\left[x_{n}-d\left(x_{n}\right), x_{n}+d\left(x_{n}\right)\right], \quad n \in N^{\prime}$.

(2) $\Delta_{n+1}^{-}=\Delta_{n}^{+}$if $n \geq 1 ; \quad \Delta_{n-1}^{+}=\Delta_{n}^{-}$if $n \leq-1$.

(3) $\Delta_{1}^{-}=\Delta_{-1}^{+}=x ; \quad \bigcup_{n \neq 0} \Delta_{n}=R$.

Lemma 5.2. For every $x \in R$ there exists an $R(x)$-covering of $R$.

Proof. Let $\mu(t)=t-d(t)-x$. Then $\mu(x)=-d(x)<0$. Let us verify the $\mu\left(t_{0}\right)>0$ for some $t_{0}>x$. Assume the contrary. Then $\mu(t)=t-d(t)-x \leq 0$ for all $t>x$. From this, by the definition of $d(t),(2.8)$ and (2.4), we get a contradiction:

$$
1=\int_{t-d(t)}^{t+d(t)} \frac{d \xi}{r(\xi) h(\xi)}>\int_{x}^{t} \frac{d \xi}{r(\xi) h(\xi)} \geq \frac{1}{2} \int_{x}^{t} \frac{d \xi}{r(\xi) \rho(\xi)} \rightarrow \infty \text { as } t \rightarrow \infty .
$$

Hence $\mu\left(t_{1}\right)=0$ for some $t_{1}>x$, and we set $\Delta_{1}=\left[t_{1}-d\left(t_{1}\right), t_{1}+d\left(t_{1}\right)\right]$. Similarly, we construct $\Delta_{n}, n \geq 2$. Let us verify that $\bigcup_{n \geq 1}^{\infty} \Delta_{n}=[x, \infty)$. If this is not the case, then there exists $z$ such that $x_{n}+d\left(x_{n}\right)<z$ for all $n \geq 1$. The sequence $\left\{x_{n}\right\}_{n=1}^{\infty}$ is monotone increasing and bounded and, therefore, it converges to some $x_{0}$. Obviously, $\infty>z-x>2 \sum_{n=1}^{\infty} d\left(x_{n}\right)$, therefore, $d\left(x_{n}\right) \rightarrow 0$ as $n \rightarrow \infty$. Since $d(x)$ is continuous, one has $d\left(x_{0}\right)=0$, a contradiction to Lemma 2.3. Similarly, one can construct the covering to the left from $x$.

Remark. Assertions similar to Lemma 5.2 were used by M. Otelbaev (see [7]).

Lemma 5.3. Suppose that (1.2) and (2.1) hold. Then,

$$
\sup _{x \in R} T(x) \leq 1, \quad T(x) \stackrel{\text { def }}{=} \int_{-\infty}^{\infty} q(t) G(x, t) d t, \quad x \in R .
$$

Proof. From (2.2) it follows that for $x \in R$ :

$$
r(x) v^{\prime}(x) \geq \int_{-\infty}^{x} q(\xi) v(\xi) d \xi, \quad r(x)\left|u^{\prime}(x)\right| \geq \int_{x}^{\infty} q(\xi) u(\xi) d \xi .
$$


Now using (5.6) and once more (2.2) we get

$$
\begin{aligned}
1 & =r(x)\left[v^{\prime}(x) u(x)-u^{\prime}(x) v(x)\right] \\
& \geq u(x) \int_{-\infty}^{x} q(\xi) v(\xi) d \xi+v(x) \int_{x}^{\infty} q(\xi) u(\xi) d \xi=T(x) .
\end{aligned}
$$

Lemma 5.4. Let $\mathcal{A}>0($ see (1.10)). Then

$$
H \stackrel{\text { def }}{=} \sup _{x \in R} \int_{-\infty}^{\infty} G(x, t) d t \leq \tau \mathcal{A}^{-1} .
$$

Proof. Below we use Lemmas 5.2 (and its notation), 5.1 and 5.3:

$$
\begin{aligned}
1 & \geq T(x)=u(x) \sum_{n=-\infty}^{-1} \int_{\Delta_{n}} q(t) v(t) d t+v(x) \sum_{n=1}^{\infty} \int_{\Delta_{n}} q(t) u(t) d t \\
& \geq \frac{2}{\alpha} u(x) \sum_{n=-\infty}^{-1} v\left(x_{n}\right) d\left(x_{n}\right)\left[\frac{1}{2 d\left(x_{n}\right)} \int_{\Delta_{n}} q(t) d t\right] \\
& +\frac{2}{\alpha} v(x) \sum_{n=-\infty}^{-1} u\left(x_{n}\right) d\left(x_{n}\right)\left[\frac{1}{2 d\left(x_{n}\right)} \int_{\Delta_{n}} q(t) d t\right] \\
& \geq \frac{\mathcal{A}}{\alpha}\left\{u(x) \sum_{n=-\infty}^{-1} \int_{\Delta_{n}} v(t) \frac{v\left(x_{n}\right)}{v(t)} d t+v(x) \sum_{n=1}^{\infty} \int_{\Delta_{n}} u(t) \cdot \frac{u\left(x_{n}\right)}{u(t)} d t\right\} \\
& \geq \frac{\mathcal{A}}{\alpha^{2}}\left\{u(x) \sum_{n=-\infty}^{-1} \int_{\Delta_{n}} v(t) d t+v(x) \sum_{n=1}^{\infty} \int_{\Delta_{n}} u(t) d t\right\}=\frac{\mathcal{A}}{\alpha^{2}} \int_{-\infty}^{\infty} G(x, t) d t .
\end{aligned}
$$

Lemma 5.5. Let $p \in[1, \infty]$. Consider $G: L_{p}(R) \rightarrow L_{p}(R)$ (see (1.4)). If $H<\infty$ (see (5.7)), then $\|G\|_{p \rightarrow p} \leq H$. In particular, if $\mathcal{A}>0$ (see (1.10), then $\|G\|_{p \rightarrow p} \leq$ $\tau \mathcal{A}^{-1}$.

Proof. The operator $G: L_{1}(R) \rightarrow L_{1}(R)$ is bounded. Indeed, since $G(x, t)=$ $G(t, x)$ for $x, t \in R$, by Fubini's theorem, using $H<\infty$, we get

$$
\begin{aligned}
\|(G f)\|_{1} & \leq \int_{-\infty}^{\infty}\left(\int_{-\infty}^{\infty} G(x, t)|f(t)| d t\right) d x \\
& =\int_{-\infty}^{\infty}|f(t)|\left(\int_{-\infty}^{\infty} G(x, t) d x\right) d t \leq H\|f\|_{1} .
\end{aligned}
$$

Hence, since $G(x, t)=G(t, x)$, the operator $G: L_{\infty}(R) \rightarrow L_{\infty}(R)$ is also bounded, $\|G\|_{\infty \rightarrow \infty} \leq H$. It remains to apply the Riesz-Torin theorem [14, Ch. XII, $\left.\S 1\right]$.

Lemma 5.6. One has $G(x, t) \leq 2 h(x), x, t \in R$.

Proof. By (2.2) and (2.8) we get

$$
G(x, t)=\rho(x)\left\{\begin{array}{ll}
v(t) v(x)^{-1}, & x \geq t, \\
u(t) u(x)^{-1}, & x \geq t,
\end{array} \leq \rho(x) \leq 2 h(x)\right.
$$


Lemma 5.7. Let $p \in[1, \infty], \quad H<\infty$ (see (5.7)) and suppose that (1.7) has no solutions $z(x) \in L_{p}(R)$ apart from $z(x) \equiv 0$. Then assertions 1$\left.)-2\right)$ from $\S 1$ hold.

Proof. Let $y=(G f)(x)$. Below we apply Hölder's inequality and Lemma 5.6:

$$
|y(x)|<\left(\int_{-\infty}^{\infty} G(x, t) d t\right)^{1 / p^{\prime}}\left(\int_{-\infty}^{\infty} G(x, t)|f(t)|^{p} d t\right)^{1 / p} \leq \tau H^{1 / p^{\prime}} h(x)^{1 / p}\|f\|_{p}
$$

Hence, for $x \in R$, the following integrals converge:

$$
\int_{-\infty}^{x} v(t) f(t) d t, \quad \int_{x}^{\infty} u(t) f(t) d t
$$

and $y(x)$ is absolutely continuous for $x \in R$. Since

$$
r(x) y^{\prime}(x)=\left(r(x) u^{\prime}(x)\right) \int_{-\infty}^{x} v(t) f(t) d t+\left(r(x) v^{\prime}(x)\right) \int_{x}^{\infty} u(t) f(t) d t
$$

the functions $\left(r(x) u^{\prime}(x)\right),\left(r(x) v^{\prime}(x)\right)$ are absolutely continuous and integrals (5.8) converge, we conclude that $r(x) y^{\prime}(x)$ is absolutely continuous and, by (2.2), one has (1.1) almost everywhere. By Lemma 5.5, $\|G\|_{p \rightarrow p} \leq H$ and, therefore, $y(x) \in$ $L_{p}(R)$, and (1.1) has no other solutions in $L_{p}(R)$.

Proof of Theorem 2.3. Below we apply Hölder's inequality, (5.5) and (5.7):

$$
\begin{aligned}
& \left\|q(x)^{1 / p} y(x)\right\|_{p}^{p}=\int_{-\infty}^{\infty} q(x)\left|\int_{-\infty}^{\infty} G(x, t)\right| f(t)|d t|^{p} d x \\
& \leq \int_{-\infty}^{\infty} q(x)\left[\int_{-\infty}^{\infty} G(x, t) d t\right]^{p / p^{\prime}}\left[\int_{-\infty}^{\infty} G(x, t)|f(t)|^{p} d t\right] d x \\
& \leq(\tau \mathcal{A})^{-p / p^{\prime}} \int_{-\infty}^{\infty}|f(t)|^{p}\left[\int_{-\infty}^{\infty} q(x) G(x, t) d x\right] d t \leq(\tau \mathcal{A})^{-p / p^{\prime}}\|f(x)\|_{p}^{p} .
\end{aligned}
$$

Proof of Corollary 2.3.1. The proof follows from Theorem 2.3.

\section{REFERENCES}

1. N. Chernyavskaya and L. Shuster, On the WKB-method, Differentsial'nye Uravneniya 25 (1989), 1826-1829. MR 90k:34065

2. - On a representation of the solution of the Sturm-Liouville equation and its applications, Differentsial'nye Uravnenia 28 (1992), 537-540. MR 93f:34088

3. __ Estimates for Green's function of the Sturm-Liouville operator, J. Diff. Eq. 111 (1994), 410-420. MR 95i:34044

4. - Asymptotic integration of a nonoscillatory second order differential equation with linear perturbation, preprint, AMS PPS \# 199508-34-001 (1995).

5. __ Estimates for the Green function of a general Sturm-Liouville operator and their application, preprint, AMS PPS \# 1130-34-002 (1997).

6. E.B. Davies and E.M. Harrell, Conformally flat Riemannian metrics, Schrödinger operators and semiclassical approximation, J. Diff. Eq. 66 (1987), 165-188. MR 88a:35061

7. K. Mynbaev and M. Otelbaev, "Weighted Functional Spaces and Differential Operator Spectrum”, Nauka, Moscow, 1988. MR 89h:46036

8. R. Oinarov, Properties of a Sturm-Liouville operator in $L_{p}$, Izvestija Akad. Nauk Kazakh. SSR 1 (1990), 43-47. MR 91k:47116

9. M. Sapenov and L. Shuster, Estimates of Green's function and theorem on separability of a Sturm-Liouville operator in $L_{p}$, Manuscript deposited at VINITI, No. 8257-B85. 
10. L. Shuster, A priori properties of solutions of a Sturm-Liouville equation and A.M. Molchanov's criterion, Math. Notes Ac. Sci. USSR 50 (1991), 746-751, Translation: Matematicheskie Zametki. MR 92m:34066

11. W.A. Steklov, Sur une méthode nouvelle pour résoudre plusiers problems sur le développement d'une fonction arbitraire en séries infinies, Compes Rendus, Paris 144 (1907), 1329-1332.

12. E.C. Titchmarsh, The Theory of Functions, Oxford, 1932.

13. E.T. Whittaker and G. N. Watson, A Course of Modern Analysis, Cambridge, 1958. MR 31:2375

14. A. Zygmund, Trigonometric Series, Cambridge, 1959. MR 21:6498

Department of Mathematics and Computer Science, Ben-Gurion University of the Negev, P.O.B. 653, Beer-Sheva, 84105, Israel

Department of Mathematics and Computer Science, Bar-Ilan University, Ramat-Gan 52900, ISRAEL 